\title{
Intracerebral adult stem cells transplantation increases brain-derived neurotrophic factor levels and protects against phencyclidine-induced social deficit in mice
}

\author{
R Barzilay ${ }^{1}$, T Ben-Zur ${ }^{1}$, O Sadan ${ }^{1}$, Z Bren ${ }^{1}$, M Taler ${ }^{2}$, N Lev ${ }^{1}$, I Tarasenko ${ }^{2}$, R Uzan ${ }^{2}$, I Gil-Ad ${ }^{2}$, E Melamed ${ }^{1}$, A Weizman ${ }^{2}$ and D Offen $^{1}$
}

Stem cell-based regenerative therapy is considered a promising cellular therapeutic approach for the patients with incurable brain diseases. Mesenchymal stem cells (MSCs) represent an attractive cell source for regenerative medicine strategies for the treatment of the diseased brain. Previous studies have shown that these cells improve behavioral deficits in animal models of neurological disorders such as Parkinson's and Huntington's diseases. In the current study, we examined the capability of intracerebral human MSCs transplantation (medial pre-frontal cortex) to prevent the social impairment displayed by mice after withdrawal from daily phencyclidine (PCP) administration $\left(10 \mathrm{mg} \mathrm{kg}^{-1}\right.$ daily for 14 days). Our results show that MSCs transplantation significantly prevented the PCP-induced social deficit, as assessed by the social preference test. In contrast, the PCP-induced social impairment was not modified by daily clozapine treatment. Tissue analysis revealed that the human MSCs survived in the mouse brain throughout the course of the experiment (23 days). Significantly increased cortical brain-derived neurotrophic factor levels were observed in the MSCs-treated group as compared with sham-operated controls. Furthermore, western blot analysis revealed that the ratio of phosphorylated Akt to Akt was significantly elevated in the MSCs-treated mice compared with the sham controls. Our results demonstrate that intracerebral transplantation of MSCs is beneficial in attenuating the social deficits induced by sub-chronic PCP administration. We suggest a novel therapeutic approach for the treatment of schizophrenia-like negative symptoms in animal models of the disorder.

Translational Psychiatry (2011) 1, e61; doi:10.1038/tp.2011.64; published online 13 December 2011

\section{Introduction}

Stem cells research is a rapidly developing field with implications that can revolutionize medicine, promising remedy to devastating diseases of the brain. ${ }^{1}$ Stem cellbased therapy could exploit one of the two strategies: (i) cell replacement, when the administered stem cells substitute the cells lost in a disease. An example for this approach could be transplantation of functional insulin-producing beta-cells for diabetes mellitus or dopamine-secreting cells for Parkinson's disease. (ii) Cell restoration, when the administered cells provide a trophic effect, protect, rescue and minimize ongoing deterioration of the tissue affected in a disease. An example of this approach would be transplanting cells that provide neurotrophic support in neurodegenerative diseases. ${ }^{2}$

Mesenchymal stem cells (MSCs) hold a potential for regenerative therapy in neurological diseases. ${ }^{1,3}$ Previous studies have shown that bone marrow MSCs express genes and proteins associated with the neural lineage, and have been shown to hold neurogenic differentiation potential in vitro. ${ }^{4-6}$ In our lab, we have shown that brain transplantation of MSCs in animal models of Parkinson's disease, Huntington's disease and multiple sclerosis results in engraftment into the brain, migration to lesioned sites and, most importantly, improvement in neuro-behavioral tests. ${ }^{2,7-12} \mathrm{~A}$ single report has recently described the efficacy of brain MSCs transplantation, also in a rat model of depression. ${ }^{13}$ In the aforementioned studies, though there is no conclusive evidence for the molecular mechanism underlying the behavioral improvement, it was shown to involve, at least in part, an increase in the availability of neurotrophic factors.

The complex pathophysiology underlying schizophrenia (SCZ) has been traditionally related to dysregulation of dopamine neurotransmission. ${ }^{14}$ Additional data suggest that SCZ is associated with glutamate NMDA receptor dysfunctions. ${ }^{15}$ This hypothesis is based on the experimental finding that agents that block NMDA receptors such as phencyclidine (PCP) and MK801 induce SCZ-like psychosis. ${ }^{16}$ Over the past few years, other molecular contributors were implicated in the disease pathophysiology, such as reduced availability of neurotrophic factors, specifically brain-derived neurotrophic factor (BDNF) in specific brain regions, ${ }^{17,18}$ activation of the immune system, ${ }^{19}$ impaired neurogenesis, ${ }^{20}$ oxidative stress ${ }^{21}$ and mitochondrial dysfunctions. ${ }^{22}$

\footnotetext{
${ }^{1}$ Laboratory of Neuroscience, Felsenstein Medical Research Center, Rabin Medical Center, Beilinson Campus, Sackler Faculty of Medicine, Tel Aviv University, Petach Tikva, Israel and ${ }^{2}$ Laboratory of Biological Psychiatry, Felsenstein Medical Research Center, Research Unit, Geha Mental Health Center, Rabin Medical Center, Sackler Faculty of Medicine, Tel Aviv University, Petach Tikva, Israel

Correspondence: Dr R Barzilay, Laboratory of Neuroscience, Felsenstein Medical Research Center, Rabin Medical Center, Beilinson Campus, Sackler Faculty of Medicine, Tel Aviv University, Petach Tikva 49100, Israel.

E-mail: barzilyr@post.tau.ac.il

Keywords: animal model; BDNF; phencyclidine; schizophrenia; social behavior; stem cells

Received 8 August 2011; accepted 23 August 2011
} 
Modeling SCZ in rodents is a challenge due to the complexity, the lack of clarity regarding the molecular processes involved in SCZ and the difficulty to relate distinct behavioral abnormalities in rodents to specific relevant symptoms in patients. ${ }^{23,24}$ However, in recent years studies have shown that specific animal models can be used as tools to investigate distinct endophenotypes of the disease. ${ }^{25}$ Subchronic PCP administration is considered to establish specific endophenotypes associated with $\mathrm{SCZ},{ }^{26}$ especially the negative symptoms. Specifically, social behavior of rodents treated with PCP is impaired while locomotor activity is preserved. $^{27}$

To date, no study has investigated the application of a stem cell-based therapeutic strategy in an animal model of SCZ-like behaviors. A single report has indicated the potential of this approach in an in vitro study, using neural stem cells to protect cultured neurons against insult induced by a specific NMDA receptor antagonist. ${ }^{28}$ In the present study, we sought to conduct a proof-of-concept experiment to explore the possible benefit of MSCs transplantation for PCP-induced impaired social behavior. We transplanted MSCs into the prefrontal cortex of mice sub-chronically treated with PCP and hypothesized that the engrafted MSCs will prevent or attenuate the development of PCP-induced impaired social behavior.

\section{Materials and methods}

The subacute PCP mouse model. A total of 54 male C57BL/6 mice aged 6 weeks (Harlan, Jerusalem, Israel) were used in this experiment. Mice were placed under $12 \mathrm{~h}$ light $/ 12 \mathrm{~h}$ dark conditions and grown in individual ventilated cages with ad libitum access to food and water. All experimental protocols were approved by the University Committee of Animal Use for Research and Education. PCP (Sigma-Aldrich, St. Lewis, MO, USA; $10 \mathrm{mg} \mathrm{kg}^{-1}$ dissolved in $0.9 \%$ normal saline) was injected subcutaneously daily for 14 days.

Cell culture and Transplantation. Human MSCs were purchased from the Cambrex Bio Science Walkersville (Walkersville, MD, USA), cultured and expanded as previously described. ${ }^{2}$ On treatment day, the cells were harvested, washed and prepared for transplantation at a concentration of 40000 cells ul $^{-1}$. Under chloral hydrate anesthesia, the mice were placed in a digital stereotactic frame (Stoelting, Wood Dale, IL, USA) and the cells $(1 \mu \mathrm{l} \mathrm{per}$ injection site) were injected bilaterally to the following coordinates (relative to the bregma and dura): anteriorposterior $+2 \mathrm{~mm}$, medial-lateral $\pm 0.4 \mathrm{~mm}$, dorsal-ventral $-3.5 \mathrm{~mm}$ at a rate of $1 \mu \mathrm{min}^{-1}$ using a Hamilton $701 \mathrm{~N}$ syringe (Sigma-Aldrich). The inserted needle was withdrawn from each location after $5 \mathrm{~min}$. Cells viability was assessed at the end of transplantation session using Trypan blue (SigmaAldrich). For cell tracking purposes, four animals in each group received injection of cells labeled with the red fluorescent marker PKH-26 (Sigma-Aldrich).

Experimental design. Transplantation took place at the first day of PCP administration. Sham surgery mice underwent

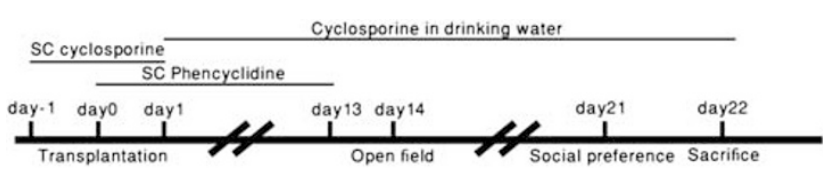

Figure 1 The study design.

identical surgical procedure without cells (saline injection only), clozapine-treated mice received daily clozapine injections ( $6 \mathrm{mg} \mathrm{kg}^{-1}$ i.p., from the first PCP administration), daily until the day of testing. The following groups were evaluated: controls (saline s.c., $n=14$ ), sham surgery PCP treated $(n=15)$, MSCs transplanted PCP treated $(n=13)$, clozapine and PCP treated $(n=12)$. In order to prevent immune response, animals received $15 \mathrm{mg} \mathrm{kg}^{-1}$ s.c. cyclosporine A (Novartis, Basel, Switzerland) for 3 days around transplantation. Thereafter, cyclosporine was added to drinking water (15 $\mathrm{mg} \mathrm{kg}^{-1}$ according to expected daily drinking volume per mouse). Behavioral tests included openfield test conducted 1 day after last PCP administration, and social preference test was conducted a week after the last PCP administration. Animals were killed 1 day following the social preference test. The experimental design is depicted in Figure 1.

Behavioral tests. Open-field test was conducted by introducing the animals into a $50-\mathrm{cm}^{2}$ arena and videotaping the spontaneous behavior of the mice for 60 min. The effects of sub-chronic PCP treatment on social behavior were assessed using the social preference test, as previously described. ${ }^{29}$ The apparatus was divided into three compartments (one central and two lateral) containing two transparent plastic cups with holes placed on either side of the arena. Before the test day, mice were habituated for two consecutive days for $20 \mathrm{~min}$, during that time the mice were free to explore the middle chamber. On the test day, the test mouse was initially habituated to the arena for $10 \mathrm{~min}$, during that time two black partitions completely covered the sides of the arena containing the cups. Thereafter, the Plexiglas partitions were removed and an unfamiliar male C57BL/6 mouse was placed in one cup (social stimulus). The other cup remained empty (inanimate stimulus). The test mouse was free to explore all the chambers and was videotaped for another $10 \mathrm{~min}$. Videos were analyzed using the Ethovision 7 software (Noldus, Wageningen, The Netheralnds), analysis allowed tracking either the center of the animal or the nose. Parameters analyzed included total time spent in each chamber; total nose pokes to the cups and total time in which the test mouse was in proximity to the cup-as a representation of social exploration. For calculation of the behavioral parameters obtained from the social preference test, we used a preference index, which is calculated as follows: if time in social chamber is $S$ and time in non social chamber is NS, then preference index equals to $(S-N S) /(S+N S)$.

Immunohistochemistry. At the end of the experiment (23 days post transplantation), eight animals of each group (four 
animals that were transplanted with the pre-labeled MSCs and four other randomly selected animals) were anesthetized with chloral hydrate and transcardially perfused with cold phosphate-buffered saline, followed by $4 \%$ paraformaldehyde in phosphate buffer. The brains were then immersed in $4 \%$ paraformaldehyde for $24 \mathrm{~h}$ at $4{ }^{\circ} \mathrm{C}$ followed by cryoprotection in $30 \%$ sucrose for an additional $48 \mathrm{~h}$. The brains were frozen in chilled 2-methylbutane (Sigma-Aldrich), stored at $-70^{\circ} \mathrm{C}$, and subsequently sectioned to slices measuring $10 \mu \mathrm{M}$. For microglial labeling, fluorescein isothiocyanate-conjugated Bandeiraea simplicifolia isolectin B4 (1:50 in phosphate-buffered saline; Sigma-Aldrich) was added for $1 \mathrm{~h}$. Detection of BDNF was conducted following 5 min antigen retrieval in 5\% SDS using rabbit anti-BDNF antibody (1:200 in blocking solution, Chemicon/Millipore, Billerica, MA, USA) followed by highly absorbed goat anti-rabbit Alexa 488 (1:500, Molecular Probes/Invitrogen, Carlsbad, CA, USA) diluted in phosphate-buffered saline. Nuclei were counterstained with 4',6-diamidino-2-phenylindole (Sigma-Aldrich). Sections were mounted with fluorescent mounting solution (Dako, Glostrup, Denmark), covered with a cover slide and sealed. Digital images were obtained with a fluorescence Olympus BX52TF microscope (Olympus, Tokyo, Japan).

Western blot. At the end of the experiment, based on proximity to the average behavioral data, four animals from each group were killed using $\mathrm{CO}_{2}$. Immediately thereafter, crude dissection of the brains was conducted and whole cortex tissues were separated and cryopreserved in $-70^{\circ} \mathrm{C}$. Consequently, tissue was thawed and total protein was produced as previously described. ${ }^{30}$ Protein concentration was determined using BCA kit (Thermo Scientific, Rockford, IL, USA). Protein samples underwent western blot analysis as described previously. ${ }^{31}$ Fifty microgram of protein was loaded in each lane. The following antibodies were employed: rabbit anti-AKT, rabbit anti-phospho-AKT (Cell Signaling Technology, Danvers, MA, USA), anti-ERK (extracellular signal-regulated kinase), anti-phospho-ERK (Santa Cruz Biotechnology, Santa Cruz, CA, USA) and anti actin (Millipore). Visualization and analysis of band intensities were performed using the Odyssey system (LI-COR, Lincoln, NE, USA).

ELISA. Quantification of BDNF levels was conducted using a BDNF specific enzyme-linked immunosorbent assay (ELISA) kit (Millipore) according to the manufacturer's instructions. Protein extracts were loaded on the ELISA plate (in quadruple samples, $25 \mu \mathrm{g}$ protein in each well). The absorbance at 450 and $570 \mathrm{~nm}$ was recorded on a Microplate Reader (Labsystems, Helsinki, Finland). Results were normalized to total amount of protein.

Statistics. Data were analyzed using SPSS 17.0. software (SPSS, Inc., Chicago, IL, USA). Comparisons between groups were performed with the two-tailed analysis of variance, with Tukey post hoc test. The results were considered significant at $P<0.05$. All results are expressed as mean \pm s.e.m.

\section{Results}

Intra-cortical transplantation of MSCs prevents the impairment of social preference exhibited in PCPtreated mice. A day after the last PCP administration, mice were observed in the open-field test and showed no difference in total locomotor behavior (Supplementary Figure 1). One week later, mice underwent the social preference test. In the first 10 min of the test in which the mouse was limited to explore the central zone, empty of the social/non social stimulus, there was no difference in the total locomotor activity of the mice (as observed by total distance moved, Figure 2a). However, after $10 \mathrm{~min}$, when allowed to explore both the chambers, that is, the chamber harboring the social stimulus and the chamber harboring the non-social stimulus, we found significant differences between the treatment groups in the preference towards the social stimulus.

Control saline-injected mice showed significant preference towards the social stimulus. In contrast, PCPtreated mice, which received sham surgery, did not show significant preference for the social stimulus, whereas the PCP-treated mice transplanted with MSCs showed significant preference towards the social stimulus. Daily clozapine administration to the PCP-treated mice did not alter the impaired social preference. Analysis of the social preference indices revealed that the same patterns of preference were registered for the total time spent in chambers (Figure 2b), total number of nose pokes towards the stimuli (Figure 2c) and total time in close proximity to the stimuli (Figure 2d).

The transplanted human MSCs survive in the mouse brain along the experiment without prominent immune reaction. To evaluate the effect of $\mathrm{PCP}$ and the insult of intra-cortical injection on global inflammatory state in the brain (regardless of cell transplantation), we stained the sham-treated brains with the fluorescein isothiocyanateconjugated microglia marker IB4 and did not detect positive staining at the end of the experiment (Figure 3a). Upon analysis of the MSCs-treated mouse brains, we first attempted to locate the transplanted human MSCs. Fluorescent microscopy analysis revealed that the transplanted MSCs, pre-labeled with the red fluorescent dye $\mathrm{PKH}-26$, were detected in the cortex near the site of transplantation (Figure 3b). We did not find any $\mathrm{PKH}-26-$ labeled cells in other brain areas such as the subventricular zone or the hippocampus. To assess the possibility of immune rejection in the brains following xenograft transplantation, we stained the sections harboring the transplanted cells. Intact $\mathrm{PKH}-26$-positive cells indicated MSCS survival throughout the experiment. We did detect some microglia staining overlap the transplanted cells staining, suggesting that immune response was present only to some extent (Figures $3 c-e$ ).

Increased levels of BDNF in the cortex following MSC transplantation. Protein lysates of dissected cortical tissues from PCP-treated mice were analyzed by ELISA. 

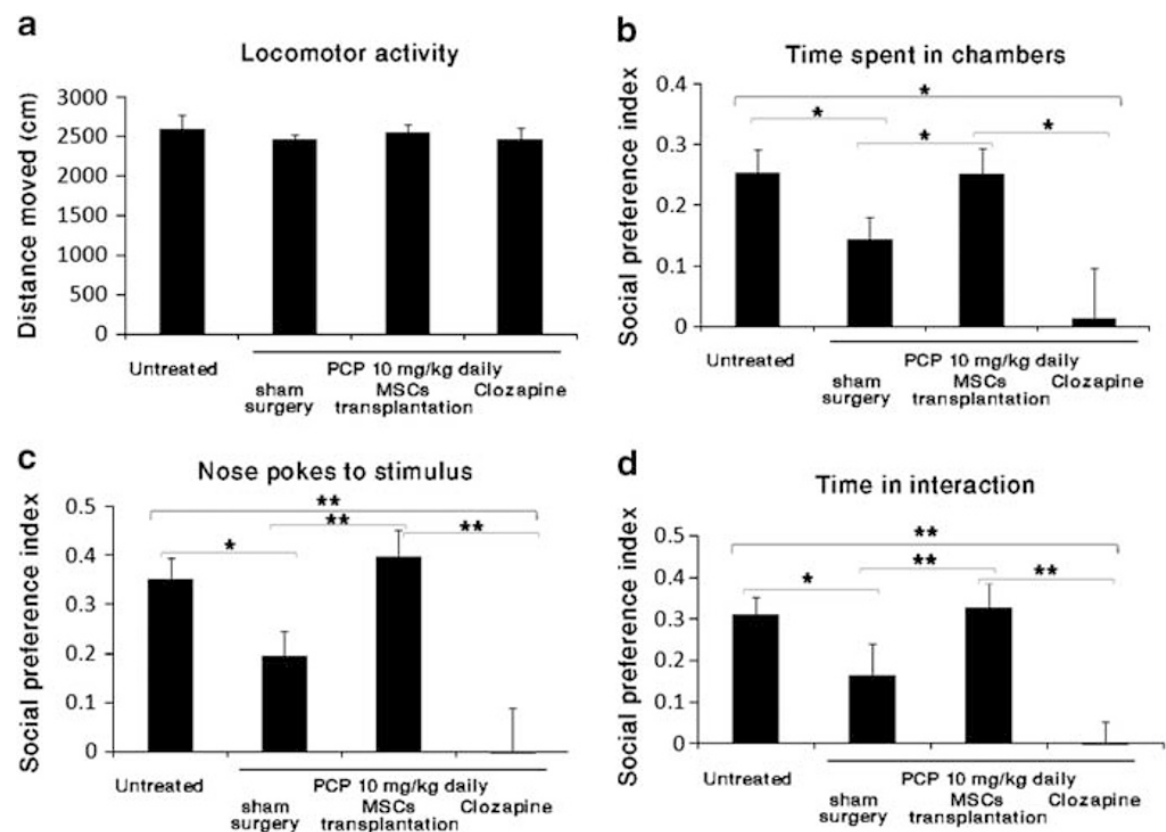

Figure 2 Social preference test. (a) Total distance moved in the $10 \mathrm{~min}$ before the exploration of the lateral compartments harboring the social and inanimate stimuli. (b-d) Preference index calculated in $10 \mathrm{~min}$ at which the test mouse was free to explore all the chambers. (b) Preference index of time spent in chamber. (c) Preference index of frequency of nose pokes to the social stimulus. (d) Preference index in social exploration. Results are displayed as mean + s.e.m. Preference index was calculated as follows: if social is $S$ and non-social is NS, then index $=(S-N S) /(S+N S) .{ }^{*} P<0.05,{ }^{* \star} P<0.01$. MSCs, mesenchymal stem cells; $P C P$, phencyclidine.
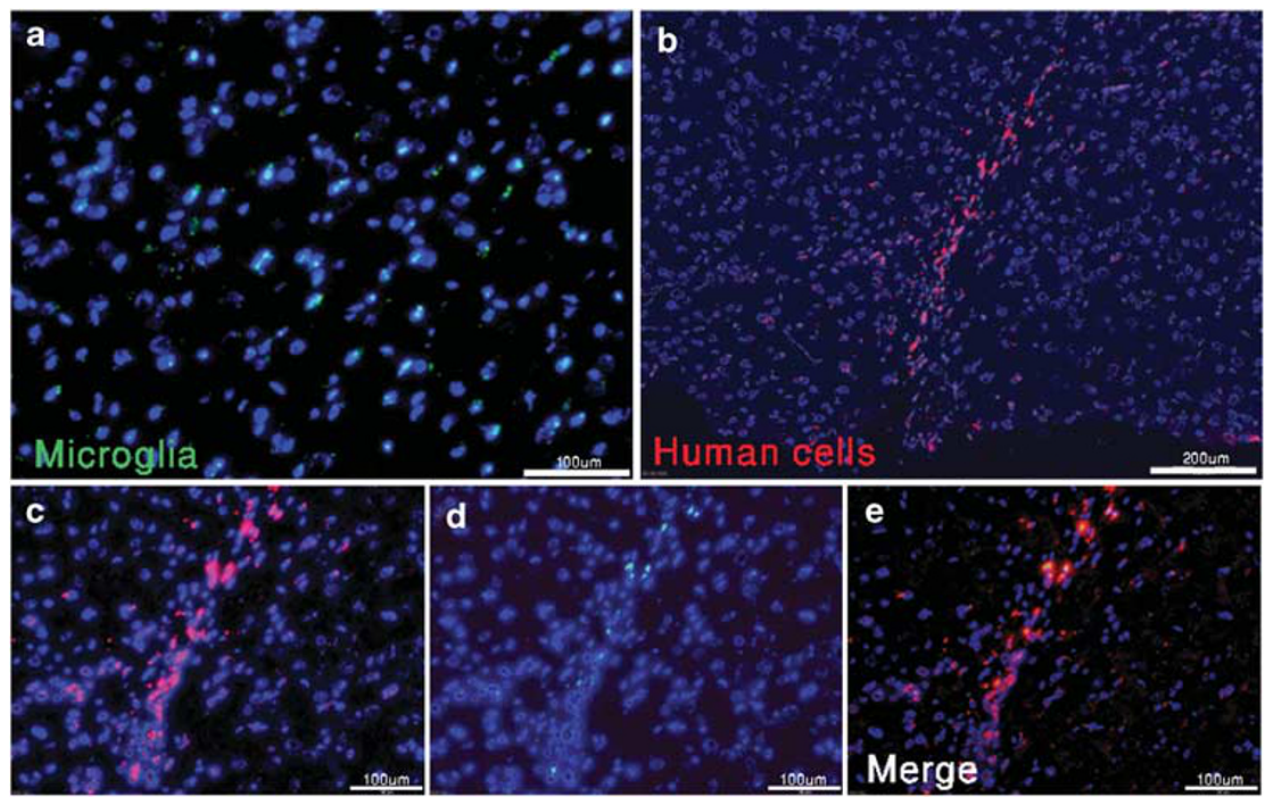

Figure 3 Immunohistochemistry of cortical brain slices. (a) Green signal is microglia stained with fluorescein isothiocyanate-conjugated IB4 in the brain of mouse treated with PCP who underwent sham transplantation. (b) Red cells are human MSCs pre-labeled with PKH-26. (c-e) Evaluation of rejection using merged photo of PKH-26-labeled MSCs and microglia staining. IB4, Bandeiraea simplicifolia isolectin B4; MSCs, mesenchymal stem cells; PCP, phencyclidine.

We found $20.53 \%$ higher BDNF levels in the cortex of MSCs-transplanted mice in comparison with mice injected with saline $(P<0.05$, Figure $4 a)$. BDNF levels in the MSCs-treated group was $32.25 \% \quad(P<0.01)$ higher compared with the clozapine-treated group. Of note, no changes in total BDNF levels were observed in the hippocampal tissues of the mouse groups (data not shown). Immunohistochemistry study using anti-BDNF antibodies in the brain sections revealed that the transplanted MSCs expressed BDNF. However, the majority of the BDNF-positive cells were mouse cells located in the transplanted area (Figures $4 \mathrm{~b}$ and $\mathrm{c}$ ). 

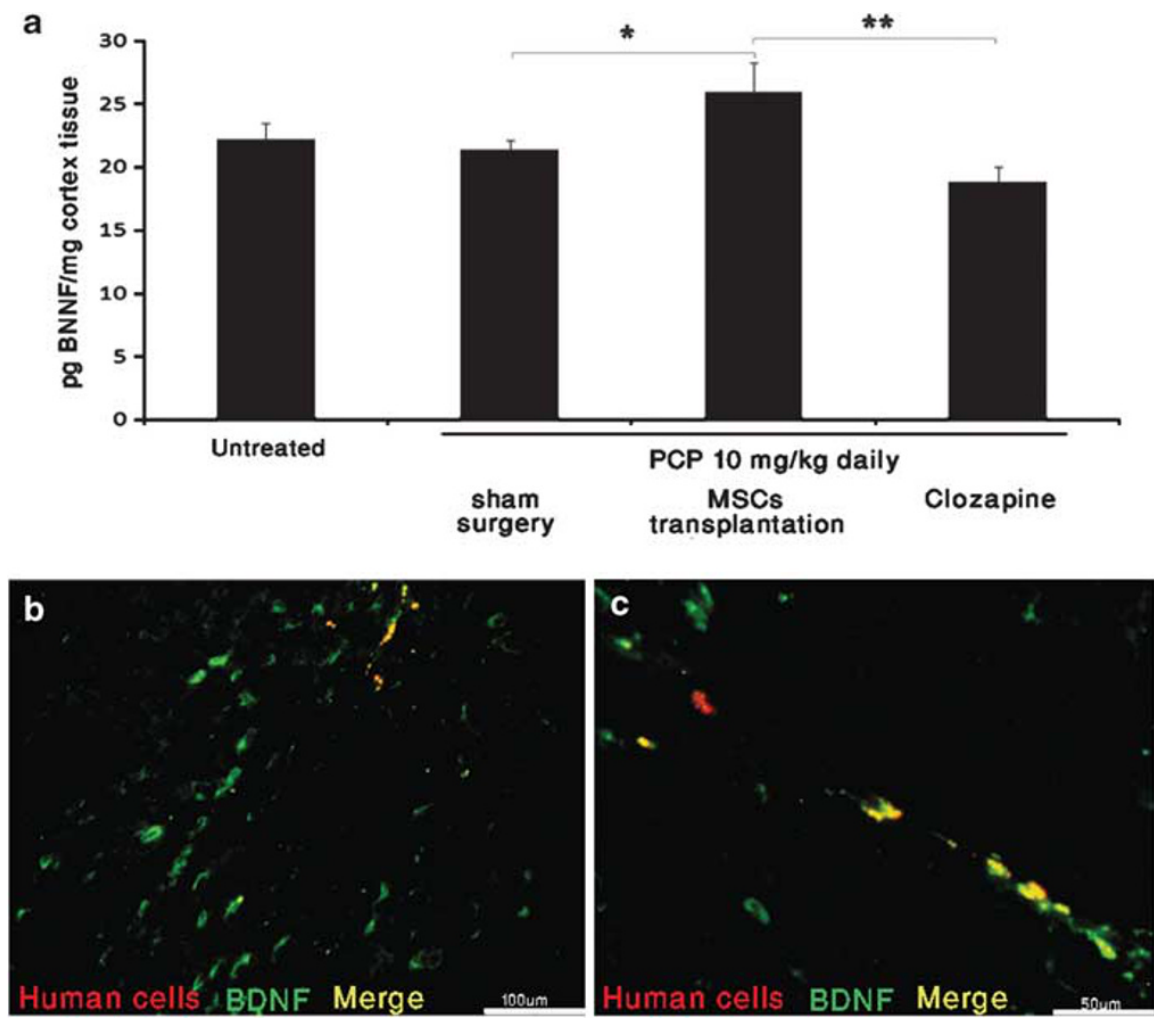

Figure 4 BDNF levels in the cortex. (a) Quantification of BDNF levels using ELISA, results represent picogram BDNF measured normalized to milligram cortex tissue $\left({ }^{\star} P<0.05,{ }^{\star \star} P<0.01\right)$. (b, c) Immunohistochemistry against BDNF in cortical brain slices of mice transplanted with MSCs (red, PKH pre-labeled human cells; green, BDNF signal; yellow, merge). BDNF, brain-derived neurotrophic factor; ELISA, enzyme-linked immunosorbent assay; MSCs, mesenchymal stem cells; PCP, phencyclidine.
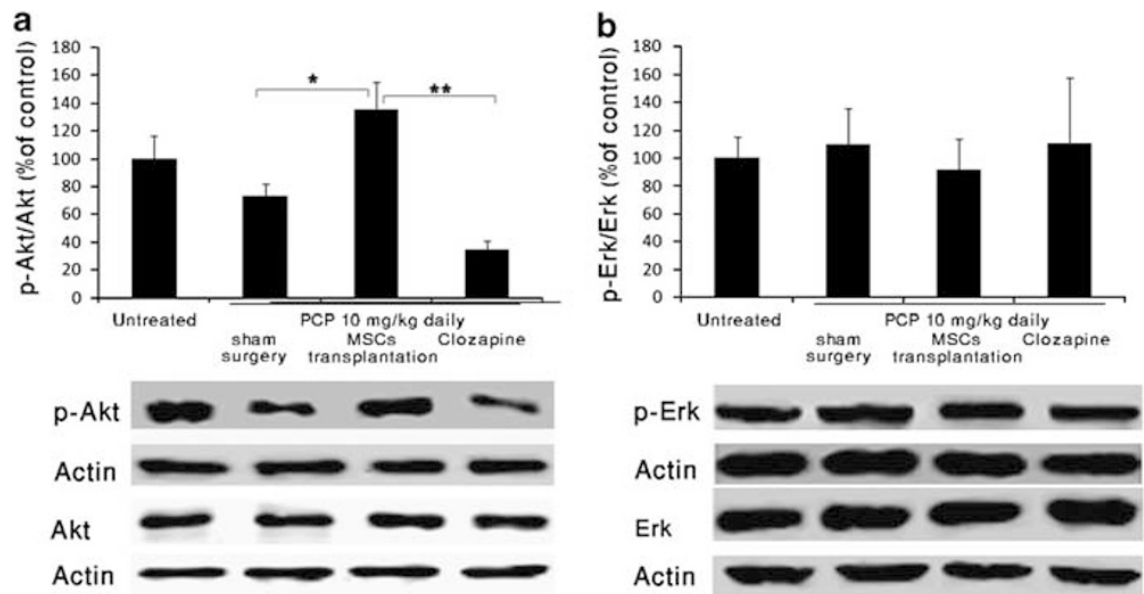

Figure 5 Western blot analysis of phosphorylated AKT (a) and ERK (b) in cortical lysates. Results represent ratio of phosphorylated protein to total protein-each was normalized to actin. The ratio obtained in untreated controls (saline instead of PCP (phencyclidine) and no cell/clozapine treatment) is represented as $100 \%$. ${ }^{*} P<0.05$, ${ }^{\star *} P<0.01$

MSCs transplantation into the cortex resulted in increased $\mathbf{p}$-AKT/total AKT ratio. To evaluate the possible effect of MSCs transplantation on specific molecular pathways associated with PCP-induced insult, we analyzed phosphorylated AKT and ERK levels by western blot. Analysis of the ratios of phosphorylated ERK to total ERK (Figure 5b) did not reveal significant differences between the groups. In contrast, we found significant difference in the levels of AKT phosphorylation in the examined protein lysates of the cortex (Figure 5a), namely, the percent of phosphorylated-AKT from total AKT was significantly higher in the cortex of the MSCs-treated mice as compared with the PCP sham surgery group (1.85-fold, $P<0.05)$. AKT phosphorylation ratio in MSCs-treated group 
was also significantly higher compared with the PCPclozapine group (3.82-fold, $P<0.01$ ).

\section{Discussion}

In this study, we present a novel approach for the treatment of an animal model of SCZ-like social impairment. In the current proof-of-concept study, we specifically show that the social deficit behavior induced by sub-chronic administration of PCP can be prevented by intra-cortical transplantation of MSCs, at variance from clozapine that failed to prevent the social deficit. The notion of applying MSCs-based therapeutic approach to an animal model of SCZ stems from the two complementing details: (1) the frustrating deadlock of antipsychotics in the treatment of negative symptoms of SCZ. ${ }^{32}$ (2) The accumulating body of evidence suggesting the benefit of MSCs-based therapeutic strategies for brain diseases. $^{33,34}$

Current treatments for SCZ are aimed mainly at antagonizing dopamine and other neurotransmitter receptors, and yield relatively limited success in the treatment of SCZ-related negative symptoms and cognitive deficits. Importantly, current antipsychotic medications rely upon the rationale of over-active dopaminergic activity or other dysregulated neurotransmitter systems in the brain. Therefore, antipsychotics are efficient in controlling the positive symptoms, which are strongly associated with post-synaptic monoamine receptors over-activity. ${ }^{14}$ Recent studies suggest that the pathophysiology underlying SCZ may also involves processes associated with neurodegeneration. ${ }^{21,22,35-37}$ For these aspects of the disease, current antipsychotic therapy offers limited resolution. Intra-brain MSCs transplantation has been shown to influence specific processes relevant to the pathophysiology of neuropsychiatric diseases. Those include the effect of MSCs on the (i) brain immune status, ${ }^{38}$ (ii) neurotrophic factor availability ${ }^{39,40}$ and (iii) neurogenesis. ${ }^{41,42}$

Designing the study, we sought to utilize one of the most established rodent model of SCZ-negative symptoms, namely, the subacute PCP model. ${ }^{43}$ We chose the social preference test as it presents a relatively straightforward paradigm of measuring sociability and was recently validated in $\mathrm{C} 57 / \mathrm{BL}$ mice. ${ }^{27}$ We performed cell transplantation concomitantly with the first dose of PCP, aiming to provide the transplanted cells the optimal chances of protection against the iatrogenic insult. Transplantation was aimed at the anatomical site, which is assumed to be involved in the social insult induced by the PCP, namely, the prefrontal cortex. ${ }^{44,45}$

We included clozapine-treated mice as a comparator group owing to a report which had shown that clozapine treatment could reverse some of the social deficits induced by subchronic PCP treatment. ${ }^{46}$ The fact that clozapine did not reverse the social deficits in our study may be explained by the differences in the behavioral test, the mouse strain or the treatment regimen employed in our study.

We chose to transplant human MSCs as they are more readily cultured, better characterized, not tumorogenic and not contaminated by hematopoietic cells in comparison with mouse-derived MSCs. ${ }^{47}$ Importantly, our previous experience with transplantation into various models of neurodegenerative diseases has shown that human MSCs survive in the brain and exert some beneficial effects. ${ }^{2,8,10}$ This choice faced us with the challenge of immune rejection and the need to use cyclosporine. In order to minimize the possible effects of cyclosporine on the social behavior, ${ }^{48}$ all the groups, including the control, received identical cyclosporine treatment. Evidently, the intensity of immunosuppression we employed was sufficient to allow the human MSCs to survive in the brain along the whole experiment period, 23 days, with only limited immune rejection.

The behavioral results of the social preference test seemed to confirm our hypothesis that MSCs could act to protect against the social deficit induced by the PCP administration. As it is known that MSCs modulate immune response in the $\mathrm{CNS}^{38}$ we first evaluated whether an immune process/ inflammation occurred following the PCP insult. To that end, we used the staining for microglia and found that no inflammation was present in the brains following the PCP treatment.

Neurotrophic factor availability, specifically BDNF, is postulated to have a key role in many aspects of brain development, maintenance and function, including the formation of synapses and neural circuits. ${ }^{49,50}$ Several studies have shown that dysregulation of BDNF expression in specific brain regions may be involved in the pathophysiology of various neuropsychiatric disorders, including SCZ. ${ }^{51,52}$ Of note, BDNF has emerged as a key factor in the development and maintenance of social behavior. ${ }^{53,54}$ Importantly, BDNF mRNA levels were recently reported to be downregulated in the prefrontal cortex of female rats sub-chronically treated with PCP. ${ }^{55}$ MSCs are known to enhance neurotrophic factor availability, either by direct secretion by the engrafted cells ${ }^{2}$ or by enhancement of endogenous levels through in situ interaction with the host brain cells. ${ }^{40}$ On the basis of the above mentioned data, we postulated that transplantation of MSCs into the cortex of mice sub-chronically treated with PCP, could increase BDNF levels adjacent to the transplantation site, and counteract some of the negative behavioral effects of the PCP. Notwithstanding, we cannot rule out that other soluble factors that are increased following administration of MSCs could also be involved in the positive behavioral outcome of MSCs intra-cortical transplantation. ${ }^{56}$

Quantitative ELISA analysis of BDNF levels in the cortex extracts of PCP-injected mice indeed demonstrated higher BDNF levels in the MSCs-treated group compared with the sham-operated or clozapine-treated groups. The relatively low number of transplanted cells (only 40000 cells per cortex) together with the immunohistochemical analysis of BDNF implies that although some BDNF was secreted from the transplanted human MSCs, most BDNF originated from the endogenous mouse brain cells. This explanation is in context with a recent report in a stroke rat model that has shown that MSCs transplantation induces a therapeutic response through the induction of the host astrocytes to upregulate the levels of neurotrophic factors. ${ }^{40}$

Recently it was shown that, in vitro, acute exposure of neurons to PCP results in rapid death through apoptosis. ${ }^{57}$ Importantly, this study has demonstrated that the insult induced by PCP could be prevented following the addition of recombinant BDNF. It was suggested that the protective effect of BDNF is associated with the activation of the 
phosphatidylinositol 3-kinase/AKT and the ERK pathways. Interestingly, the only report to date to examine stem cell protective effect on cultured neurons following NMDA receptor blockade showed that the protection occurs via AKT/ERK signal regulation. ${ }^{28}$ Our data show that MSCs transplantation results in higher phosphorylated AKT/total AKT ratio as compared with the sham-operated or clozapinetreated mice. We could not detect significant differences in the phosphorylated ERK ratios or in the total levels of cleaved caspase (data not shown). This could be explained by the fact that we killed the animals 10 days following the last PCP dose, while the in vitro study mentioned above showed that PCP. induced apoptosis occurs within hours after the exposure. ${ }^{57}$

Our study serves as a pioneer proof-of-concept for cellbased therapeutic strategies for SCZ animal models. To our knowledge, this is the first report of stem cell transplantation study in an animal model of SCZ. Evidently, future studies would have to face the challenge of enlarging the battery of behavioral tests representing several SCZ-related endophenotypes/dimensions. We have shown that MSCs transplantation can protect against the PCP insult, however, future studies should be performed in an attempt to explore the possibility of reversing an already established damage (that is, transplant cells after the last PCP dose), or to try and use stem cell-based approaches in relevant transgenic mouse models of SCZ. Another prospect that would have to be explored in the future is the optimal site of transplantation, that is, evaluation of effects of MSCs in other brain sites affected in SCZ and PCP abuse, such as the hippocampus.

To conclude, we showed that intra-cortical MSCs transplantation protected against the social deficit induced by subchronic PCP administration. Analysis of the mouse brains showed higher levels of BDNF in the MSCs-treated mouse cortex and elevated ration of $\mathrm{p}$-AKT/AKT compared with the sham-treated controls. Interestingly, BDNF and $p$-AKT were recently reported to protect neuronal culture against $\mathrm{PCP}$ insult in vitro. Our study suggests that MSCs transplantation might be considered, at least at the experimental laboratory level, as a putative strategy for the treatment of some SCZrelated endophenotypes/dimensions, such as negative symptoms and cognitive deficits.

\section{Conflict of interest}

The authors declare no conflict of interest.

Acknowledgements. This research was supported by the National Institute for Psychobiology in Israel.

1. Lindvall O, Kokaia Z. Stem cells for the treatment of neurological disorders. Nature 2006; 441: 1094-1096.

2. Sadan O, Bahat-Stromza M, Barhum Y, Levy YS, Pisnevsky A, Peretz $\mathrm{H}$ et al. Protective effects of neurotrophic factors secreting cells in a 6-OHDA rat model of Parkinson disease. Stem Cells Dev 2009; 18: 1179-1190.

3. Barzilay R, Levy SY, Melamed E, Offen D. Adult stem cells for neuronal repair. Isr Med Assoc J 2006; 8: 61-66.

4. Tondreau T, Lagneaux L, Dejeneffe M, Massy M, Mortier C, Delforge A et al. Bone marrow derived mesenchymal stem cells already express specific neural proteins before any differentiation. Differentiation 2004; 72: 319-326.
5. Blondheim NR, Levy YS, Ben-Zur T, Burshtein A, Cherlow T, Kan I et al. Human mesenchymal stem cells express neural genes, suggesting a neural predisposition. Stem Cells Dev 2006; 15: 141-164.

6. Deng J, Petersen BE, Steindler DA, Jorgensen ML, Laywell ED. Mesenchymal stem cells spontaneously express neural proteins in culture and are neurogenic after transplantation. Stem Cells 2006; 24: 1054-1064.

7. Bahat-Stroomza M, Barhum Y, Levy YS, Karpov O, Bulvik S, Melamed E et al. Induction of adult human bone marrow mesenchymal stromal cells into functional astrocyte-like cells: potential for restorative treatment in Parkinson's disease. J Mol Neurosc 2009; 39: 199-210

8. Barhum Y, Gai Castro S, Bahat-Stroomza M, Barzilay R, Melamed E, Offen D. Intracerebroventricular transplantation of human mesenchymal stem cells induced to secrete neurotrophic factors attenuates clinical symptoms in a mouse model of multiple sclerosis. J Mol Neurosc 2010; 41: 129-137.

9. Hellmann MA, Panet H, Barhum Y, Melamed E, Offen D. Increased survival and migration of engrafted mesenchymal bone marrow stem cells in 6-hydroxydopamine-lesioned rodents. Neurosci Lett 2006; 395: 124-128.

10. Levy YS, Bahat-Stroomza M, Barzilay R, Burshtein A, Bulvik S, Barhum $Y$ et al. Regenerative effect of neural-induced human mesenchymal stromal cells in rat models of Parkinson's disease. Cytotherapy 2008; 10: 340-352.

11. Offen D, Barhum Y, Levy YS, Burshtein A, Cherlow T, Melamed E. Intrastriatal transplantation of mouse bone marrow-derived stem cells improves motor behavior in a mouse model of Parkinson's disease. J Neural Transm Suppl 2007; 72: 133-143.

12. Sadan O, Shemesh N, Barzilay R, Bahat-Stromza M, Melamed E, Cohen Y et al. Migration of neurotrophic factors-secreting mesenchymal stem cells towards a quinolinic acid lesion as viewed by MRI. Stem Cells 2008; 26: 2542-2551.

13. Tfilin M, Sudai E, Merenlender A, Gispan I, Yadid G, Turgeman G. Mesenchymal stem cells increase hippocampal neurogenesis and counteract depressive-like behavior. $\mathrm{Mol}$ Psychiatr 2010; 15: 1164-1175.

14. Lieberman JA, Bymaster FP, Meltzer HY, Deutch AY, Duncan GE, Marx CE et al. Antipsychotic drugs: comparison in animal models of efficacy, neurotransmitter regulation, and neuroprotection. Pharmacol Rev 2008; 60: 358-403.

15. Kantrowitz J, Javitt D. Thinking glutamatergically: changing concepts of schizophrenia based upon changing neurochemical models. Clin Schizophr Relat Psychoses 2010; 4: 189-200.

16. Coyle JT. The glutamatergic dysfunction hypothesis for schizophrenia. Harv Rev Psychiatry 1996; 3: 241-253.

17. Durany N, Michel T, Zochling R, Boissl KW, Cruz-Sanchez FF, Riederer P et al. Brainderived neurotrophic factor and neurotrophin 3 in schizophrenic psychoses. Schizophr Res 2001; 52: 79-86.

18. Weickert CS, Hyde TM, Lipska BK, Herman MM, Weinberger DR, Kleinman JE. Reduced brain-derived neurotrophic factor in prefrontal cortex of patients with schizophrenia. $\mathrm{Mol}$ Psychiatr 2003; 8: 592-610.

19. Arion D, Unger T, Lewis DA, Levitt $P$, Mirnics K. Molecular evidence for increased expression of genes related to immune and chaperone function in the prefrontal cortex in schizophrenia. Biol Psychiat 2007; 62: 711-721.

20. Eisch AJ, Cameron HA, Encinas JM, Meltzer LA, Ming GL, Overstreet-Wadiche LS. Adult neurogenesis, mental health, and mental illness: hope or hype? J Neurosci 2008; 28: $11785-11791$.

21. Ng F, Berk M, Dean O, Bush Al. Oxidative stress in psychiatric disorders: evidence base and therapeutic implications. Int J Neuropsychoph 2008; 11: 851-876.

22. Rezin G, Amboni G, Zugno A, Quevedo J, Streck E. Mitochondrial dysfunction and psychiatric disorders. Neurochem Res 2009; 34: 1021-1029.

23. Lipska BK, Weinberger DR. To model a psychiatric disorder in animals: schizophrenia as a reality test. Neuropsychopharmacol 2000; 23: 223-239.

24. Nestler EJ, Hyman SE. Animal models of neuropsychiatric disorders. Nat Neurosci 2010; 13: $1161-1169$.

25. Amann LC, Gandal MJ, Halene TB, Ehrlichman RS, White SL, McCarren HS et al. Mouse behavioral endophenotypes for schizophrenia. Brain Res Bul 2010; 83: 147-161.

26. Nabeshima T, Mouri A, Murai R, Noda Y. Animal model of schizophrenia: dysfunction of NMDA receptor-signaling in mice following withdrawal from repeated administration of phencyclidine. Ann NY Acad Sci 2006; 1086: 160-168.

27. Brigman JL, Ihne J, Sakisda LM, Bussey TJ, Holmes A. Effects of subchronic phencyclidine (PCP) treatment on social behaviors, and operant discrimination and reversal learning in C57BL/6J mice. Front Behav Neurosci 2009; 3: 2.

28. Ono $\mathrm{T}$, Hashimoto $\mathrm{E}$, Ukai $\mathrm{W}$, Ishii $\mathrm{T}$, Saito $\mathrm{T}$. The role of neural stem cells for in vitro models of schizophrenia: neuroprotection via Akt/ERK signal regulation. Schizophr Res 2010; 122: 239-247.

29. Nadler JJ, Moy SS, Dold G, Simmons N, Perez A, Young NB et al. Automated apparatus for quantitation of social approach behaviors in mice. Genes Brain Behav 2004; 3: 303-314.

30. Grunbaum-Novak N, Taler M, Gil-Ad I, Weizman A, Cohen H, Weizman R. Relationship between antidepressants and IGF-1 system in the brain: possible role in cognition. Eur Neuropsychopharm 2008; 18: 431-438.

31. Taler M, Bar M, Korob I, Lomnitski L, Baharav E, Grunbaum-Novak N et al. Evidence for an inhibitory immunomodulatory effect of selected antidepressants on rat splenocytes: possible relevance to depression and hyperactive-immune disorders. Int Immunopharmacol 2008; 8: 526-533. 
32. Abbott A. Schizophrenia: the drug deadlock. Nature 2010; 468: 158-159.

33. Sadan $\mathrm{O}$, Melamed E, Offen D. Bone-marrow-derived mesenchymal stem cell therapy for neurodegenerative diseases. Expert Opin Biol Th 2009; 9: 1487-1497.

34. Sensebe L, Krampera M, Schrezenmeier H, Bourin P, Giordano R. Mesenchymal stem cells for clinical application. Vox Sanguinis 2010; 98: 93-107.

35. Csernansky JG. Neurodegeneration in schizophrenia: evidence from in vivo neuroimaging studies. ScientificWorldJournal 2007; 7: 135-143.

36. Jarskog LF, Miyamoto S, Lieberman JA. Schizophrenia: new pathological insights and therapies. Annu Rev Med 2007; 58: 49-61.

37. Do KQ, Cabungcal JH, Frank A, Steullet $P$, Cuenod M. Redox dysregulation, neurodevelopment, and schizophrenia. Curr Opin Neurobiol 2009; 19: 220-230.

38. Kassis I, Grigoriadis N, Gowda-Kurkalli B, Mizrachi-Kol R, Ben-Hur T, Slavin S et al. Neuroprotection and immunomodulation with mesenchymal stem cells in chronic experimental autoimmune encephalomyelitis. Arch Neurol 2008; 65: 753-761.

39. Rodrigues Hell RC, Silva Costa MM, Goes AM, Oliveira ALR. Local injection of BDNF producing mesenchymal stem cells increases neuronal survival and synaptic stability following ventral root avulsion. Neurobiol Dis 2009; 33: 290-300.

40. Shen LH, Li Y, Chopp M. Astrocytic endogenous glial cell derived neurotrophic factor production is enhanced by bone marrow stromal cell transplantation in the ischemic boundary zone after stroke in adult rats. Glia 2010; 58: 1074-1081.

41. Kan I, Barhum Y, Melamed E, Offen D. Mesenchymal stem cells stimulate endogenous neurogenesis in the subventricular zone of adult mice. Stem Cell Rev 2011; 7: 404-412.

42. Bao X, Wei J, Feng M, Lu S, Li G, Dou W et al. Transplantation of human bone marrowderived mesenchymal stem cells promotes behavioral recovery and endogenous neurogenesis after cerebral ischemia in rats. Brain Res 2011; 1367: 103-113.

43. Seillier A, Giuffrida A. Evaluation of NMDA receptor models of schizophrenia: divergences in the behavioral effects of sub-chronic PCP and MK-801. Behav Brain Res 2009; 204 410-415.

44. Schwabe K, Klein S, Koch M. Behavioural effects of neonatal lesions of the medial prefrontal cortex and subchronic pubertal treatment with phencyclidine of adult rats. Behav Brain Res 2006; 168: 150-160.

45. Wood JN. Social cognition and the prefrontal cortex. Behav Cogn Neurosci Rev 2003; 2 : 97-114.

46. Qiao $\mathrm{H}$, Noda $\mathrm{Y}$, Kamei $\mathrm{H}$, Nagai T, Furukawa $\mathrm{H}$, Miura $\mathrm{H}$ et al. Clozapine, but not haloperidol, reverses social behavior deficit in mice during withdrawal from chronic phencyclidine treatment. Neuroreport 2001; 12: 11-16.
47. Prockop DJ. Repair of tissues by adult stem/progenitor cells (MSCs): controversies, myths, and changing paradigms. Mol Ther 2009; 17: 939-946.

48. Sato Y, Takayanagi Y, Onaka T, Kobayashi E. Impact of cyclosporine upon emotional and social behavior in mice. Transplantation 2007; 83: 1365-1370.

49. Greenberg ME, Xu B, Lu B, Hempstead BL. New insights in the biology of BDNF synthesis and release: implications in CNS function. J Neurosci 2009; 29: 12764-12767.

50. Cohen-Cory S, Kidane AH, Shirkey NJ, Marshak S. Brain-derived neurotrophic factor and the development of structural neuronal connectivity. Dev Neurobiol 2010; 70 271-288.

51. Martinowich K, Manji H, Lu B. New insights into BDNF function in depression and anxiety. Nat Neurosci 2007; 10: 1089-1093.

52. Angelucci F, Brene S, Mathe AA. BDNF in schizophrenia, depression and corresponding animal models. Mol Psychiatr 2005; 10: 345-352.

53. Schaevitz LR, Moriuchi JM, Nag N, Mellot TJ, Berger-Sweeney J. Cognitive and socia functions and growth factors in a mouse model of Rett syndrome. Physiol Behav2010; 100: 255-263.

54. Broad KD, Mimmack ML, Keverne EB, Kendrick KM. Increased BDNF and trk-B mRNA expression in cortical and limbic regions following formation of a social recognition memory. Eur J Neurosci 2002; 16: 2166-2174.

55. Snigdha S, Neill J, McLean S, Shemar G, Cruise L, Shahid M et al. Phencyclidine (PCP)induced disruption in cognitive performance is gender-specific and associated with a reduction in brain-derived neurotrophic factor (BDNF) in specific regions of the female rat brain. J Mol Neurosci 2011; 43: 337-345.

56. Prockop DJ, Kota DJ, Bazhanov N, Reger RL. Evolving paradigms for repair of tissues by adult stem/progenitor cells (MSCs). J Cell Mol Med 2010; 14: 2190-2199.

57. Xia Y, Wang CZ, Liu J, Anastasio NC, Johnson KM. Brain-derived neurotrophic factor prevents phencyclidine-induced apoptosis in developing brain by parallel activation of both the ERK and PI-3K/Akt pathways. Neuropharmacol 2010; 58: 330-336.

Translational Psychiatry is an open-access journal published by Nature Publishing Group. This work is licensed under the Creative Commons Attribution-Noncommercial-No Derivative Works 3.0 Unported License. To view a copy of this license, visit http://creativecommons.org/licenses/by-nc-nd/3.0/

\section{Supplementary Information accompanies the paper on the Translational Psychiatry website (http://www.nature.com/tp)}

